\title{
C3-BIOECONOMY
}

Circular and Sustainable Bioeconomy

\section{El comercio del carbón vegetal y su transitar hacia la bioeconomía en}

\section{México}

\author{
Noel Carrillo Ávila ${ }^{1}$, Adán Hernández Hernández ${ }^{2}$ y Blanca Patricia Castellanos- \\ Potenciano $^{3}$
}

Autor de Correspondencia: castellanos.blanca@inifap.gob.mx

\section{Resumen:}

El consumo de carbón vegetal a nivel global se ha incrementado entre un 3 y $4 \%$ de forma anual en los últimos años. Su uso en México, es un factor socio-cultural por lo que su consumo podría llegar hasta las 700 mil toneladas cada año, con una perspectiva de crecimiento de 360 mil toneladas al 2030, lo que implicaría disponer de más de dos millones de metros cúbicos de madera para tal fin. En general, no existe un proceso estandarizado para la producción de carbón vegetal para una calidad homogénea, su elaboración se realiza de manera artesanal con diferentes especies forestales y en hornos de tierra rudimentarios, con técnicas que no brindan altos rendimientos. Además, su comercialización está llena de opacidades que limitan cuantificar los verdaderos volúmenes que se comercializan. Así, la elaboración y comercialización del carbón podría constituir una amenaza para el futuro de los recursos maderables. Dada esta problemática, el objetivo del texto es plantear la situación problemática que se observa entorno al comercio del carbón en México y expone la necesidad de investigación que permita transitar en la economía circular a través del manejo sustentable, por medio de una conversión energética alta (más carbón con menos madera), plantaciones exprofeso y el uso eficiente de residuos para fines energéticos, con estrategias tecnológicas que mitiguen los impactos ambientales negativos.

Palabras clave: recursos-forestales, economía-circular, sustentabilidad

\section{The charcoal trade and its transition to the bioeconomy in Mexico}

\author{
Noel Carrillo Ávila ${ }^{1}$, Adán Hernández Hernández ${ }^{2}$ y Blanca Patricia Castellanos-
}

\section{Potenciano ${ }^{3}$}

\begin{abstract}
:
Global charcoal consumption has increased between 3 and $4 \%$ annually in recent years. Its use in Mexico is a socio-cultural factor, so its consumption could reach up to 700 thousand tons each year, with a growth perspective of 360 thousand tons by 2030 , which would imply having more than two million cubic meters. of wood for this purpose. In general, there is no standardized process for the production of charcoal for a homogeneous quality, its elaboration is carried out in a

1 Instituto Nacional de Investigaciones Forestales, Agrícolas y Pecuarias (INIFAP) Centro de Investigación Golfo Centro, Campo Experimental San Martinito, Puebla. carrillo.noel@inifap.gob.mx.

2 Instituto Nacional de Investigaciones Forestales, Agrícolas y Pecuarias (INIFAP) Centro de Investigación Regional Pacifico Sur, Campo Experimental Valles Centrales de Oaxaca, hernandez.adan@inifap.gob.mx.

3 Instituto Nacional de Investigaciones Forestales, Agrícolas y Pecuarias (INIFAP) Centro de Investigación Regional Pacifico Sur, Campo Experimental Valles Centrales de Oaxaca. castellanos.blanca@inifap.gob.mx. ORCID 0000-0002-7605-4991.
\end{abstract}

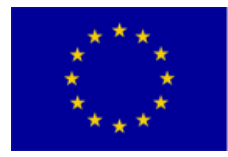

UNIÓN EUROPEA

PROYECTO COFINANCIADO POR EL FONDO EUROPEO DE DESARROLIO REGIONAO Una manera de hacer Europa

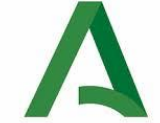

Junta de Andalucía
Andalucía

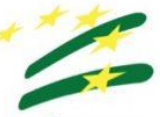

se mueve con Europa 
traditional way with different forest species and in rudimentary earth ovens, with techniques that do not provide high yields. In addition, its commercialization is full of opacities that limit the quantification of the true volumes that are commercialized. Thus, the processing and marketing of charcoal could constitute a threat to the future of timber resources. Given this problem, the objective of the text is to present the problematic situation that is observed around the coal trade in Mexico and exposes the need for research that allows moving in the circular economy through sustainable management, through high energy conversion (more coal with less wood), exprofeso plantations and the efficient use of waste for energy purposes, with technological strategies that mitigate negative environmental impacts

Keywords: forest-resources, circular-economy, sustainability

\section{LA RED INNOVAGRO Y LA BIOECONOMÍA}

Hasta el presente año, el volumen y el comercio mundial del carbón mostraron una tendencia creciente, lo que afecta negativamente tanto al medio ambiente como a la seguridad del suministro de carbón (Wang et al., 2021). Si bien el carbón vegetal sigue desempeñando un papel vital en la estructura energética de países en Latinoamérica, en esta dinámica también se insertan los principales productores a nivel global como, países de África, India, Japón y China (Figura 1) (Sribna et al., 2019, FAOSTAT, 2020).

Figura 1. Los diez principales países productores de carbón vegetal.

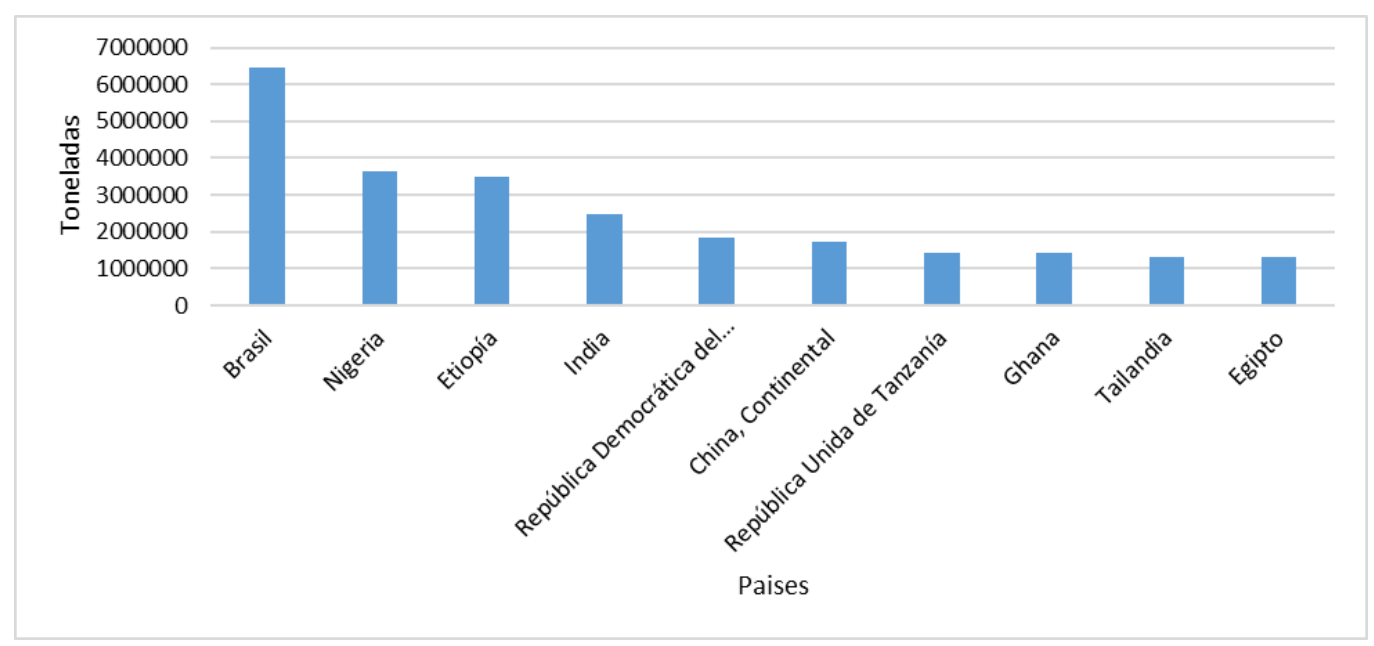

Fuente: FAOSTAT, 2020.

En lo general, la degradación forestal asociada a la producción de carbón vegetal es difícil de monitorear y comúnmente se pasa por alto y está subrepresentada en los cambios de la cubierta forestal y en las estimaciones de emisiones de carbono en el mundo (Sedano et al., 2016). 
En México la forma de producir y distribuir el carbón vegetal es muy peculiar, ya que no hay patrones definidos para su elaboración y consumo. Hay varias formas o técnicas para su producción y, la forma como se realiza el comercio y se distribuye se encuentra dentro en la informalidad, desde su primera venta, hasta su uso final (Camou-Guerrero, et al., 2014; Morales et al., 2018). Así, cuantificar el consumo de carbón es complicado, debido a distintos motivos como que, i) la inexistencia de registros oficiales, ya que una parte de éste combustible proviene de la colecta realizada por los habitantes para su abastecimiento, y la otra proviene del comercio informal (Nabukalu et al., 2019); ii) existen variaciones importantes del consumo en México, dadas las condiciones socioculturales y climáticas que determinan la vegetación predominante y su disponibilidad (Díaz et al., 2020) ; iii) la información disponible no permite desagregar de manera específica los usos finales de este dendroenergético, ya que tiene propósitos simultáneos (cocción, calefacción y calentamiento); además que, iv) existe un consumo paralelo en combustibles (en específico, del gas licuado de petróleo o gas LP), en complemento con el carbón vegetal (Morales, et al., 2018).

Por lo expuesto, este texto tiene el objetivo de plantear la problemática que se observa entorno al comercio del carbón en México y expone la necesidad de investigación que permita transitar en la economía circular a través del manejo sustentable.

Ante el escenario anteriormente citado, se plantea la necesidad de superar la antigua visión del sector agropecuario como generador de bienes primarios y ver a la agricultura como la industria de la biomasa y el actor central de los nuevos tiempos en sociedades que aspiran a ser más eficientes en el uso de los recursos naturales y menos dependientes de los recursos fósiles. Para ello se requieren modelos productivos climáticamente responsables, en los que la bioeconomía expresa en buena medida esa visión transformadora para promover el desarrollo agrícola sostenible e inteligente, partiendo de la amplia base de recursos biológicos que poseemos (FAO. 2019). 


\section{DESARROLLO}

\subsection{Tendencias de producción mundial del carbón vegetal}

El carbón vegetal es un biocombustible que se produce como consecuencia de una combustión incompleta de la madera, por medio de un proceso de pirolisis (Hernández-Hernández, et al., 2020). El carbón es un mejor combustible, ya que presenta la gran ventaja frente a la madera (como la leña o las astillas), debido a que no es atacable por microorganismos al ser un material prácticamente inerte (Barbaran, 2019).

Según la Organización de las Naciones Unidas para la Agricultura y la Alimentación (FAO), más del $50 \%$ de la madera de los bosques en todo el mundo se utiliza para la producción de energía (Nabukalu \& Gieré, 2019). En todo el mundo, el carbón vegetal ha persistido como recurso energético y el incremento en su producción se ha mantenido desde 1993 al 2020 (Figura 2) (FAOSTAT, 2020).

Figura 2. Producción mundial de carbón vegetal a nivel mundial.

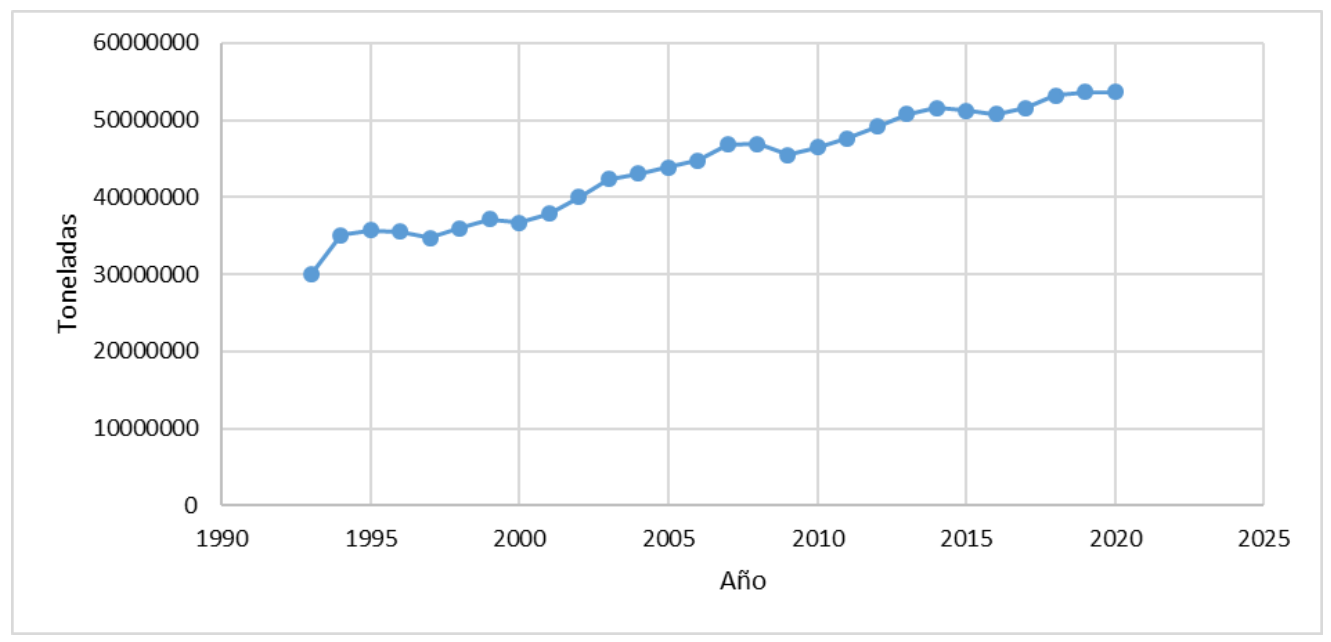

Fuente: FAOSTAT, 2020.

La producción y consumo de carbón vegetal en México, es un factor sociocultural, que ha cobrado relevancia en las últimas décadas, sin embargo, se ha producido y utilizado como combustible para cocinar alimentos desde la edad de piedra y para producir instrumentos metálicos desde la edad de bronce (Rios, et. al., 2017). 
En la actualidad, se utiliza ampliamente en hogares urbanos y rurales para cocer y asar, ya que produce poco humo y tiene un alto valor calorífico. En la Unión Europea, el uso del carbón vegetal es en lo general "recreativo" (días de campo, barbacoas), por lo que importa el producto de países como Alemania, Polonia, Suiza, España, Italia, Noruega, Dinamarca, Países Bajos, Ucrania, República Checa y Bélgica (Haag et al., 2020).

Por otra parte, en México los usos son para cocinar, calentar hogares, así como también el recreativo, siendo los principales centros de con ciudades como Monterrey, Cd. de México, Guadalajara y Puebla (Camou-Guerrero, 2014) por lo que su consumo podría llegar hasta las 700 mil toneladas cada año y la perspectiva de crecimiento podría incrementarse en 360 mil toneladas al 2030, (Serrano, et al., 2014), lo que como consecuencia implicaría disponer de más de dos millones de metros cúbicos de madera para tal fin.

\subsection{Cadena de valor de consumo}

Para la comercialización del carbón vegetal, los intermediarios han sido el medio más factible para vender la producción. Esto, ha limitado que los productores accedan a los mercados para comercializar directamente con el consumidor, aunado a la falta de organización de los productores y de estrategias para la comercialización. Por lo que esta dependencia hacia los intermediarios como vía de comercialización, ha ocasionado una desventaja sobre el precio para los productores, provocando una sobrevaloración en el costo del producto y durante su paso por la cadena de comercialización. De esta forma el "pago" económico es sustancialmente desproporcionado a lo largo de la cadena de valor en comparación con otros oficios (Nabukalu \& Gieré, 2019) lo que deja, una mayor ganancia económica para intermediarios o comerciantes, que a los productores quienes realizan el mayor trabajo para producir el carbón.

La actividad carbonera en México, tiene potencial para aprovechar oportunidades de comercialización en mercados extranjeros (De la Cruz, et. al., 
2020); sin embargo, la temporalidad, los problemas de organización, estandarización en la producción, comercialización y el desconocimiento de la oportunidad de mercado, provocan que la actividad se mantenga confinada a las áreas de producción y sea poco remunerable para el productor común.

En general, proceso de elaboración de carbón se realiza de manera artesanal con diferentes especies de recursos forestales y en hornos de tierra rudimentarios, utilizando técnicas con las que no se obtienen altos rendimientos (Heya, et. al, 2014; FAO, 2017; De la Cruz, et. al., 2020). Como consecuencia, se observa la falta de un proceso estandarizado para la producción de carbón vegetal, que permita una calidad más homogénea del producto.

En los países desarrollados el aumento en la demanda como combustible para la cocción de alimentos (barbacoas o parrilladas), así como en producción metalúrgica (Stassen, 2002, Dufourny, et al., 2019), el carbón vegetal ha incrementado su volumen de importación. En el mercado europeo, aunque el carbón vegetal tiene una participación significativa como producto a base de madera, aún no está cubierto por el Reglamento Europeo de la Madera (EUTR), (UE) No 995/2010, lo que limita la comprensión del flujo del carbón vegetal que se importa y comercialización en estos países, desde su origen, la calidad y destino comercial (Haag et al., 2020).

El comercio internacional de combustibles de madera (incluyendo los combustibles líquidos) ha crecido con rapidez (Faaij, 2002; Nabukalu \& Gieré, 2019) lo que se estima, está en correlación al incremento de la poblacional urbana, siendo la demanda creciente de un 3 a un $4 \%$ anual (Girard, 2002; FAO, 2017; Chiteculo et al., 2018).

\subsection{Implicaciones del consumo}

No obstante, donde existe una alta demanda de carbón vegetal hay además una fuerte presión sobre los recursos forestales, aumentando la presión sobre los problemas de deforestación (Girard, 2002; Nabukalu \& Gieré, 2019). Así, se debe prestar atención al creciente consumo de carbón vegetal asociado a los procesos de urbanización y la producción empresarial, ya que muchas de las 
actuales prácticas y técnicas de fabricación de carbón vegetal están fijados en la valía económica y no, en la ecológica y social (Trossero, 2002).

En el territorio mexicano, la investigación sobre los impactos ecológicos y socioeconómicos sobre la producción y comercialización del carbón, recibe poca atención (Manzón, 2015). Así, el uso de la biomasa forestal para la elaboración de carbón podría constituir una amenaza para el futuro de los recursos maderables, en especial en ciertas áreas con fuerte demanda y donde se carece de políticas y normas adecuadas para el ordenamiento territorial, con base a investigación científica y los planes de manejo forestal (Chidumayo et al., 2013)

Por ello, es preciso adecuar prácticas de gestión, supervisión y control forestal; garantizando que el crecimiento en el uso del carbón vegetal no repercuta gravemente sobre las zonas forestales que suministran a los centros de consumo, mediante la garantía de la biomasa y la reducción de la deforestación (Doggart et al., 2017).

La prohibición de la producción y comercialización del carbón vegetal, ha resultado según algunos estudios, contraproducente: no reduce la producción y si promueve el clandestinaje e impide el control y registro (Eniola y Odebode, 2018). Por lo que resurge la necesidad de dirigir esfuerzos para aumentar la calidad y disponibilidad estadística del carbón vegetal, por medio de tecnologías que brinden una conversión energética más eficiente para evitar desperdicios y hacer un uso sustentable de la biomasa y transitar hacia un sector forestal bioeconómico (Bahdon et al., 2002).

El aprovechamiento de los recursos forestales ha sido objeto de un intenso debate en los últimos años tanto en el ámbito nacional como internacional, donde se ha expresado la enorme preocupación por la acelerada deforestación de los bosques, lo que ha llevado a tomar medidas unilaterales al prohibir el comercio de maderas y productos forestales. 
Otras estrategias, se han orientado en el manejo sostenible de los bosques para reducir los impactos ecológicos y sociales. Sin embargo, el impacto de la actividad carbonera sobre los recursos naturales puede dejar un saldo ecológico negativo en las zonas dedicadas a esta actividad, cuando no existen programas de manejo forestal para la producción (Oladegy, et al., 2020). Aabeyir et al. (2016), expone que el impacto de la producción de carbón vegetal a menudo es demasiado generalizado y la perdida de la cobertura vegetal, de los sitios cosechados es un problema de manejo de los bosques después de la cosecha y no el impacto de la producción de carbón vegetal perse.

La producción de carbón vegetal en México es una actividad arraigada en el medio rural, considerada como una actividad de subsistencia marginal y poco o nada rentable para los productores, quienes terminan utilizando la mano de obra familiar y cuyos costos llegan a ser mayores que los beneficios que obtienen por la venta del producto, ya que cuentan con muy pocas opciones disponibles para poder subsistir (Sánchez, 1995). Al contrario de países como África subsahariana donde el carbón vegetal tiene el potencial de apoyar a millones de medios de vida rurales y urbanos a través de la generación de ingresos, proporcionando flujos financieros urbanos-rurales y contribuyendo a la economía nacional; p. ej. en Malawi, el sector del carbón vegetal aporta aproximadamente $\$ 40$ millones, aproximadamente el $0,5 \%$ del PIB nacional (Smith et al., 2017).

Sin embargo, los comerciantes y los responsables finales como, las empresas procesadoras, tienen las máximas ganancias por el producto y no han previsto un aprovechamiento sustentable, ya que anteponen la maximización de ganancias a costa del impacto ecológico (Ramos, 1999).

Así, se hace evidente la urgencia de adoptar políticas adecuadas para asegurar la efectividad de la sostenibilidad, ya que la producción tradicional de carbón vegetal puede apoyar el sustento de los pequeños productores tradicionales, siempre que se planee la disponibilidad de recursos y, por lo tanto, su sostenibilidad a largo plazo (Smith et al., 2017), y así contribuir con el logro de 
los objetivos de desarrollo sostenible en los países en desarrollo (Villazón, et al., 2018).

En México la Comisión Nacional Forestal (CONAFOR), a través del Programa Apoyos para el Desarrollo Forestal Sustentable 2021 ha promovido la transferencia de tecnología, a través de las capacitaciones en la elaboración de carbón para orientar el aprovechamiento de forma sustentable de los recursos, vigilar las quemas para conseguir carbón de alta entereza y maximizar las características deseables como la densidad, tamaño, peso, dureza y químicamente puro, para que los volátiles no presenten chispa o humos. Además, que realizan recomendaciones para que durante el manejo no absorba demasiada humedad y el producto mantenga los contenidos energéticos máximos, contribuyendo con un menor desperdicio, en los centros de producción o de comercialización, para contribuir en garantizar la economía de los productores (CONAFOR,2021).

\section{CONCLUSIONES}

Las alternativas actuales para mitigar la situación problemática del comercio y producción de carbón vegetal en México, debe involucrar el uso de paquetes tecnológicos que contribuyan en, i) maximizar la conversión energética de la biomasa y se reduzca el desperdicio del recurso, ii) acompañamiento técnico para la transferencia de la tecnología; así como, iii) distribución del producto y desarrollo de estrategias de venta.

Es importante señalar que no se cuenta con información exacta que permita cuantificar la producción de carbón vegetal en México, derivado de la irregularidad de su comercio. Así como de la escasa investigación científica entorno a esta problemática. En consecuencia, surge la necesidad de proponer acciones que promuevan regular el comercio, reduciendo el intermediarismo a través de la capacitación a productores; promoviendo la incorporación sólida de los subsistemas natural y social (fundamentales de cualquier plan de manejo sustentable), y de sus interacciones. Evidenciando la necesidad en investigación 
para caracterizar la cadena de producción en las regiones carboneras del país, así como el diseño y la implementación de prácticas y programas de manejo de recursos, fundamentales para transitar hacia una economía carbonera circular y reducir el impacto negativo de los ecosistemas.

Como parte de la economía circular es necesario eficientar la producción de forma sustentable, por medio de una conversión energética alta (lograr más carbón con menos madera), promover plantaciones exprofeso y el uso eficiente de residuos para fines energéticos, a través de la promoción de estrategias tecnológicas que mitiguen los impactos ambientales negativos.

Por eso, es importante reducir la producción de carbón vegetal de forma desordenada que implique el deterioro ecológico. Por lo que se requiere incrementar la profesionalización el sector con: organización, difusión tecnológica, plantaciones con intención, así como la promoción del uso de residuos para fines energéticos que contribuyan en la incorporación de la producción y comercio del carbón vegetal en la bioeconomía.

\section{REFERENCIAS}

AABEYIR, R., ADU-BREDU, S., AGYARE, W. A., y WEIR, M. J. C. (2016). Empirical evidence of the impact of commercial charcoal production on Woodland in the Forest-Savannah transition zone, Ghana. Energy for Sustainable Development, 33, 84-95. doi: https://doi.org/10.1016/j.esd.2016.03.005

BAHDON, J., BROADHEAD, J. y WHITEMAN, A. (2002). Revisión de las estimaciones de FAOSTAT relativas al combustible de Madera. Unasylva. FAO. Roma, Italia. 53 (211): 41-45.

BARBARAN, P. M. C. (2019). Caracterización de la producción y comercialización de carbón vegetal en el distrito de Manantay, Coronel Portillo - Ucayali. Universidad Nacional de Ucayali, Pucallpa, Peru. Tesis. $84 \mathrm{p}$.

CAMOU-GUERRERO, A., GHILARDI, T. M. A., SERRANO, M., ORTIZ-AVILA, T., VEGA, E., OYAMA, K. y MASERA, O. (2014). Análisis de la producción de carbón 
vegetal en la Cuenca del Lago de Cuitzeo, Michoacán. Investigación Ambiental. 6(2) 127-138.

CONAFOR, Comision Nacional Forestal (2021). Apoyos 2021: Programa apoyos para el desarrollo forestal sustentable. Gobierno de México. Acceso: 4 de junio del 2021. https://www.gob.mx/conafor/acciones-yprogramas/apoyos-conafor

CHIDUMAYO, E. N., y GUMBO, D. J. (2013). The environmental impacts of charcoal production in tropical ecosystems of the world: A synthesis. Energy for Sustainable Development, 17(2), 86-94. doi: https://doi.org/10.1016/j.esd.2012.07.004

CHITECULO, V., LOJKA, B., SUROVÝ, P., VERNER, V., PANAGIOTIDIS, D., Y WOITSCH, J. (2018). Value Chain of Charcoal Production and Implications for Forest Degradation: Case Study of Bié Province, Angola. 5(11), 113. https://doi.org/10.3390/environments5110113

DE LA CRUZ, MONTELONGO, C., HERRERA GAMBOA, j., ORTIZ SÁNCHEZ, I. A., RÍOS SAUCEDO, J. C., ROSALES SERNA, RIGOBERTO, y CARRILLO-PARRA, A. (2020). Caracterización energética del carbón vegetal producido en el Norte-Centro de México. Madera y bosques, 26(2), e262197. doi: https://doi.org/10.21829/myb.2020.2621971

DÍAZ, M. G., CORREA, A. R., y MÉNDEZ, R. M. (2020). Especies vegetales con uso combustible por comunidades rurales mexicanas. Revista Etnobiología., $18(3), 113-135$.

DOGGART, N., y MESHACK, C. (2017). The Marginalization of Sustainable Charcoal Production in the Policies of a Modernizing African Nation. 5(27). doi:10.3389/fenvs.2017.00027

DUFOURNY, VAN DE STEENE, A. L., HUMBERT G., GUIBAL, D., MARTIN, L., y BLIN, J. (2019. Influence of pyrolysis conditions and the nature of the wood on the quality of charcoal as a reducing agent. Journal of Analytical and Applied Pyrolysis, 137, 1-13. https://doi.org/10.1016/i.jaap.2018.10.013 
Eniola, P. O., y Odebode, S. O. (2018). Rural Dwellers' Perception of Effect of Charcoal Production on the Environment in Guinea Savannah Zone of Nigeria. Journal of Scientific Research and Reports, 19(1), 1-12. https://doi.org/10.9734/JSRR/2018/39422

FAAIJ, A. (2002). El comercio internacional de combustibles. Unasylva. FAO. Roma, Italia.

FAO. 2017. La transición al carbón vegetal, la ecologización de la cadena de valor del carbón vegetal para mitigar el cambio climático y mejorar los medios de vida locales. Roma, Italia. 12 p.

FAOSTAT. (2020). Producción forestal y comercio. Acceso: 13 de diciembre 2021 https://www.fao.org/faostat/es/\#data/FO/visualize

GIRARD, P. (2002). Producción y uso del carbón vegetal en África. Unasylva. FAO. Roma, Italia.

HAAG, V., ZEMKE, V. T., LEWANDROWSKI, T., ZAHNEN, J., HIRSCHBERGER, P., BICK, U., y KOCH, G. (2020). The European charcoal trade. IAWA Journal, 41 (4), 463-477. doi: https://doi.org/10.1163/22941932-bja10017

HERNÁNDEZ-HERNÁNDEZ, M., PALMA-LÓPEZ, D. J., SALGADO-GARCÍA, S., PALMA CANCINO, D. J., RINCÓN-RAMÍREZ, J. A., HIDALGO-MORENO, C. I., y CUANALO-DE LA CERDA, H. (2020). Carbón vegetal como mejorador de un Acrisol cultivado con caña de azúcar (Saccharum spp.). Agro Productividad, 13(5). https://doi.org/10.32854/agrop.vi.1607

HEYA, M. N., POURNAVAB, F. R., CARRILLO-PARRA, A., y COLIN-URIETA, S. (2014). Bioenergy potential of shrub from native species of northeastern Mexico. International journal of agricultural policy and research, 2(12), 475-483. doi: 10.15739/IJAPR.020.

MANZÓN, CHE, M. D. J. (2015). Evaluación del impacto socioeconómico de la producción del carbón vegetal en una comunidad forestal en la Península de Yucatán, México. Tesis (M.Sc), CATIE, Turrialba (Costa Rica).

MORALES, R. V., MUÑOZ, M. A. R., y DÍAZ, J. J. (2018). Producción y comercialización de la leña y el carbón en el municipio de Tequila, 
Veracruz: Hacia un análisis situacional. Medio ambiente, sustentabilidad y vulnerabilidad social. (5). 57-71 p.

NABUKALU, C., y GIERÉ, R. (2019). Charcoal as an Energy Resource: Global Trade, Production and Socioeconomic Practices Observed in Uganda. 8(4), 183. https://doi.org/10.3390/resources8040183

OLADEJI, S.O., OLOGUNWA, O.P., y TONKOLLIE, B.T. (2018). Impacto socioeconómico de la tecnología tradicional de producción de carbón vegetal en el distrito de Kpaai-condado de Bong en Liberia. Reinar. Manag. Sostener. Dev , 7 , 86-107. https://doi.org/10.5296/emsd.v7i2.11770

RAMOS, MARTINEZ, M. (1999). Producción y comercialización de carbón vegetal e la micro-región de Teopisca-San Cristóbal. Thèse pour l'obtention du master en Science du développement rural régiona, 187 p.

RÍOS, B, I. M., SANTOS C, J., y GUTIÉRREZ, A. C. (2017). Biocombustibles sólidos: una solución al calentamiento global. Revista Ciencia, 68(4), 1-17.

SÁNCHEZ, R. L. (1995). La producción de carbón vegetal en hornos fijos de ladrillo como una innovación tecnológica en México. Reporte de investigación. División de Ciencias Forestales. Universidad Autónoma Chapingo. Chapingo, México. 58 p.

SEDANO, F., SILVA, J. A., MACHOCO, R., MEQUE, C. H., SITOE, A., RIBEIRO, N., Y TUCKER, C. J. (2016). The impact of charcoal production on forest degradation: a case study in Tete, Mozambique. Environmental Research Letters, 11 (9), 094020. doi:10.1088/1748-9326/1 1/9/094020

SERRANO, M.; ARIAS-CHALICO, T.; GHIRALDI, A.; y MASERA, O. (2014). Spatial and temporal projection of fuelwood and charcoal consumption in Mexico. Energy for Sustainable Development 19: 39-46.

STASSEN, H.E. (2002). Nuevas tecnologías de producción de carbón vegetal. Unasylva. FAO. Roma, Italia. 53 (21 1): 34-35.

SMITH, H. E., HUDSON, M. D., y SCHRECKENBERG, K. (2017). Livelihood diversification: The role of charcoal production in southern Malawi. Energy 
for Sustainable Development, 36, 22-36. doi: https://doi.org/10.1016/j.esd.2016.10.001

SRIBNA, Y., TROKHYMETS, O., NOSATOV, I., y KRIUKOVA, I. (2019). The globalization of the world coal market - contradictions and trends. E3S Web Conf., 123.

TROSSERO, M.A. (2002). Dendroenergía: perspectivas del futuro. Unasylva. FAO. Roma, Italia 53 (211): 3-10.

VILLAZÓN, MONTALVÁN, R.A., DE MEDEIROS, MACHADO, M., PACHECO, R.M. PORTELA, NOGUEIRA, T. M., SILVA, DE CARVALHO PINTO, C. R., Y FANTINI, A (2019). Environmental concerns on traditional charcoal production: a global environmental impact value (GEIV) approach in the southern Brazilian context. Environ Dev Sustain 21, 3093-3119. https://doi.org/10.1007/s10668-018-0177-0

WANG, W., FAN, L., LI, Z., ZHOU, P., y CHEN, X. (2021). Measuring dynamic competitive relationship and intensity among the global coal importing trade. Applied Energy, 303, 117611 doi: https://doi.org/10.1016/j.apenergy.2021.117611 